\title{
Habilidades sociales como factor crítico en la satisfacción laboral
}

\author{
Social skills as a critical factor in job satisfaction
}

Teresa Celeste Naranjo Pinela ${ }^{1}$, Johana Verónica Espinel Guadalupe ${ }^{2}$, Junes Lady Robles Amaya ${ }^{3}$

INFORMACIÓN DEL

\section{ARTÍCULO}

Fecha de recepción: 13 de Noviembre

de 2019.

Fecha de aceptación: 19 de Diciembre de 2019.

$\bar{l}$ Doctora en Sociología, Universidad de Zaragoza, España. Docente e Investigadora de la Universidad Estatal de Milagro - Ecuador.

E-mail: tnaranjop@unemi.edu.ec

Código ORCID:

https://orcid.org/0000-0002-3938-238X

${ }^{2}$ Doctoranda en Psicología Universidad de Salamanca, España. Docente e Investigadora de la Universidad Estatal de Milagro - Ecuador.

E-mail: jespinelg@unemi.edu.ec

Código ORCID:

https://orcid.org/0000-0002-4867-2215X

${ }^{3}$ Doctoranda en Psicología Universidad de Jaén, España. Docente e Investigadora de la Universidad Estatal de Milagro - Ecuador

E-mail: jroblesa1@unemi.edu.ec

Código ORCID:

https://orcid.org/0000-0001-6809-0012

CITACIÓN: Naranjo Pinela, T. C., Espinel Guadalupe, Y. V. \& Robles Amaya J. L. (2019). Habilidades sociales como factor crítico en la satisfacción laboral. PODIUM, (36), 89-102.

https://doi.org/10.31095/podium.2019.

\section{ENLACE DOI:}

http://dx.doi.org/10.31095/podium.201 9.36 .6

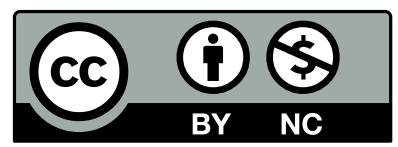

\section{Resumen}

La presente investigación tiene como objetivo establecer la relación entre las habilidades sociales y la satisfacción laboral, teniendo presente las tendencias de las organizaciones modernas, las cuales se han vuelto más competitivas y demandan de un personal con capacidades interpersonales que logren consolidar el éxito de la empresa. La metodología utilizada responde a un enfoque cuantitativo-correlacional de tipo transaccional. La muestra fue no probabilística e incluyó personas con al menos un año de experiencia laboral y que residieran en la ciudad de Milagro. Los resultados obtenidos demuestran una correlación significativa entre la edad y la forma en que se realiza la negociación en la empresa sobre los aspectos laborales.

\section{Palabras Clave:}

Habilidades sociales; satisfacción laboral; insatisfacción laboral; expresión; organizaciones; relaciones interpersonales.

\section{Clasificación JEL: J28.}

\begin{abstract}
This research aims to establish the relationship between social skills and job satisfaction, keeping in mind the trends of modern organizations, which have become more competitive and demand a staff with interpersonal skills that consolidate the success of the company. The methodology used responds to a quantitative-correlational approach of transactional type. The sample was not probabilistic and included people with at least one year of work experience and residing in the city of Milagro. The results obtained demonstrate a significant correlation between age and the way in which the negotiation is carried out in the company on labor aspects.
\end{abstract}

\section{Keywords:}

Social skills; work satisfaction; work dissatisfaction; expression; organizations; relationships.

JEL Classification: J28. 


\section{Introducción}

En la actualidad, el ser humano se encuentra condicionado al aspecto laboral de manera relativa, tal es así, que gran parte de su tiempo diario transcurre en su lugar de trabajo, lo que implica una permanente relación con el resto de individuos que se desenvuelven en este mismo ámbito. Ante aquello, es necesario que cada una de las personas sepan relacionarse y disponer de manera efectiva de un conjunto de habilidades sociales (HS) con la finalidad de evitar conflictos que deterioran el clima laboral (CL). Sin embargo, en un tiempo donde las personas se dedican más al uso de tecnologías, el tema de habilidades sociales y la inteligencia emocional no están bien desarrolladas, convirtiéndose en un reto para quienes administran los recursos humanos de las organizaciones (Berman y West, 2008). Para Patrício Do Amaral, Maia Pinto, y Bezerra de Medeiros (2015) el estudio de las habilidades sociales es fundamental, ya que permite entender las variantes de conductas que se producen en el individuo cuando es expuesto a una serie de factores que inciden en su estado de confort. A partir de aquello, el autor considera pertinente que el sujeto evidencie habilidades sociales, que establezca una relación efectiva con los demás; es decir la capacidad para escuchar, expresar sus emociones, sentimientos, ideas y trabajar colaborativamente con la convicción de crear un ambiente de complacencia que de manera relativa incide en su satisfacción laboral. De la misma manera, Flores Mamaní et al (2016) aseguran que el desempeño laboral, así como la satisfacción exige del conocimiento y dominio de las habilidades sociales, factor elemental que permite al profesional generar relaciones interpersonales saludables. A partir de aquello se destaca que, dentro del ámbito administrativo, gran parte de los estudios se basan en los aspectos básicos que giran en torno al bienestar del personal colaborativo, por lo que enfatizan en la satisfacción laboral y la capacidad que tienen éstos para establecer vínculos afectivos y de trabajo con el resto de elementos que integran la organización. Por tanto, la calidad de estas genera una oportunidad de crecimiento, así como de trabajo eficaz. En esta misma línea, Eceiza, Arrieta, y Goñi (2008) enfatizan en el abordaje del estudio de las habilidades sociales y su incidencia en la satisfacción laboral, por considerar que dentro de los ámbitos de trabajo se ventilan una serie de conductas sociales que tienden a incidir en el clima organizacional cuando éstas llegan a ser conflictivas y dominantes. Es por ello que, Moreno et al (2014) expresan que el éxito de las organizaciones se cimienta especialmente en la eficiencia de las HS que presenta su personal colaborativo, ya que estas son incidentes de manera proporcional con la SL, por lo que su relación afecta al funcionamiento de la estructura interna y gestión administrativa inherente a la organización. Es por esta razón, que el presente artículo enfatiza la necesidad de establecer la relación entre las habilidades sociales y la satisfacción laboral de un grupo de trabajadores de diferentes sectores de la ciudad de 
Milagro, provincia del Guayas, Ecuador.

\section{Revisión de literatura}

Las habilidades sociales, son consideradas un conjunto de conductas y capacidades de tipo social, que son aprendidas por los individuos y que se manifiestan en situaciones de tipo interpersonal (Eceiza, Arrieta, y Goñi, 2008). En este sentido, el aporte de García Esparza y Méndez Sánchez (2017) como resultado de su investigación sobre HS, deja entrever que éstas deben ser vistas como un grupo de estrategias o nociones sociales adquiridas por las personas para resolver situaciones de forma efectiva, eficiente y estructurada. Así también, Zavala Berbena, Valadez Sierra, y Vargas Vivero (2008) a través de su investigación referente a la inteligencia emocional y habilidades sociales en adolescentes con alta aceptación social, demuestran que las HS son patrones de conductas con características observables, aprendidas y utilizadas en los intercambios dentro de un contexto social con la finalidad de obtener fines concretos. Según lo expresado por Rodríguez Matamoros, Cacheiro González, y Gil Pascual (2014) en su estudio sobre el desarrollo de las habilidades sociales, concuerdan, al referirse a éstas como el conjunto de destrezas que facilitan el desenvolvimiento social elemental o básico de un individuo, además de incluir los niveles mínimos de cortesía alineados a las normativas sociales $u$ organizacionales permisibles. Por esta razón, se enfatiza en la presencia de varios elementos sustanciales, entre los que se encuentran la comunicación, vínculos interpersonales, expresión de intereses propios y modos de agradecimientos; para de este modo entablar un diálogo que facilite comprender las necesidades de los demás. A partir de aquello, es pertinente que las organizaciones tomen en consideración que la incorporación de nuevas tecnologías exige de los colaboradores el desarrollo de competencias profesionales que les permitan desarrollarse a pesar de los cambios y es por eso que las habilidades sociales se convierten en un mecanismo de apoyo para que las empresas sean más saludables (Moreno et al, 2014). Sin embargo, son muchos los factores que inciden en dicho éxito, donde los componentes de uno u otro son más complejos y deben ser atendidos de manera eficiente, ante aquello Ferris, Treadway y Perrewé (2007) evidencian que "la carencia de las habilidades sociales, nivel de competitividad y eficiencia no tendrían un recorrido exitoso en la satisfacción laboral de los trabajadores" (pág. 292), por tal motivo se tiene que priorizar el atender de manera efectiva aquellos aspectos que favorezcan a la creación de conductas sociales positivas.

Desde un enfoque particular, existen individuos que no logran relacionarse de manera constructiva con el resto de compañeros debido a diversas situaciones, las mismas que van desde la excesiva permisividad, agresividad, intransigencia, carencia de comunicación, dificultad al expresar sus sentimientos, problemas para escuchar a los demás, 
egocentrismo, entre otros, situación que denota una dificultad en sus habilidades sociales que puede provocar momentos desagradables en el ámbito familiar como laboral (Del Prette et al, 2012). Bajo este contexto, Haz Vidal y Fierro Ulloa (2017) destacan a la satisfacción laboral como aquellas experiencias de la persona dentro de una organización, la que es transformada en base a la percepción que éste le brinde por medio de su componente emocional. A partir de aquello, los autores consideran que para llegar a un análisis de la satisfacción laboral es necesario tomar en consideración diversas dimensiones, de las cuales se pueden priorizar los compañeros, la función del líder, el salario, las posibilidades de ascenso o logro profesional, así como la relevancia de las tareas que realiza, demostrando la vinculación de la satisfacción laboral con las habilidades sociales.

Al momento de conceptualizar sobre habilidades sociales desde la perspectiva psicosocial, Ovejero (1990) la define como "un conjunto de destrezas sociales, especialmente psicosociales que deben ser entrenadas y mejoradas" (pág. 93). Sin embargo, existe una controversia al tratar de establecer una definición exacta de las mismas, por lo que resulta dificultoso fundamentar que éstas incluyan la presencia de múltiples conductas, así como su dependencia de un contexto social, el cual resulta muy variable. En este sentido, el criterio de los autores citados converge, en que las habilidades sociales logran relacionarse de manera directa con una multiplicidad de conductas propias del individuo, que actúa dentro de un contexto interpersonal que lo induce a la expresión de sus sentimientos, actitudes, deseos, así como opiniones propias de la situación en la que se encuentra. Cornelius y Faire (2017) señalan que: "las habilidades sociales se presentan como un conjunto de conductas aprendidas, socialmente aceptadas y que, a su vez, posibilitan su interacción con las demás personas" (pág. 86), por tanto, dichas habilidades permiten que la persona logre alcanzar sus metas deseadas, por lo que se debe observar su naturaleza cognitiva y afectiva no directamente observable por medio de un proceso de evaluación, interpretación $\mathrm{y}$ debido entrenamiento que debe estar en consonancia con el contexto social.

Por otra parte, Caballo (2007) expone que las habilidades sociales son "un conjunto de conductas, identificables, aprendidas que emplean los individuos en las situaciones interpersonales para obtener o mantener el reforzamiento de su ambiente" (pág. 6). De la misma manera, Pujol-Cols y Dabos (2018) afirman que este tipo de habilidad es "la de buscar, mantener o mejorar el reforzamiento en una situación interpersonal a través de la expresión de sentimientos o deseos cuando esa expresión se arriesga a la pérdida de reforzamiento o incluso al castigo" (pág. 98).

Tapia y Cubo (2017) en su estudio sobre Habilidades sociales relevantes: percepciones de múltiples actores educativos, mencionan que: "dentro del ámbito educativo, las habilidades sociales juegan un rol significativo en el desempeño 
docente" (pág. 2), por lo que muchas investigaciones consideran que la calidad del profesor y el liderazgo directivo se presentan como las variables que determinan el peso sobre los resultados del aprendizaje de los estudiantes, sumándose a ello sus habilidades sociales por la frecuente interacción con diversos grupos de personas.

Caballo (1983) se ubica entre quienes mencionan el interés de las organizaciones por atender de manera eficiente el desarrollo de las habilidades sociales dentro del ámbito laboral. En este sentido, se hace más fácil identificar cuál de ellas posee nuestro personal colaborativo y, en qué aspectos les toca trabajar. Debido a esto el autor menciona un conjunto de aspectos que forman parte de las habilidades sociales, entre las que se encuentran: la iniciativa, comunicación, empatía, capacidad de trabajo en equipo, flexibilidad, asertividad, eficacia y eficiencia, las mismas que un individuo las puede poseer en mayor o menor medida, pero lo más importante se centra en la forma en que se puede mejorar si se logra plantear un trabajo específico en ellas.

Corredor et al (2017) sostienen que uno de los problemas para el desarrollo adecuado de las habilidades sociales, se encuentra sujeto al contexto donde se desenvuelve la persona (p. 481), por lo que este tipo de habilidad debe ser considerada dentro de un marco cultural, así como la presencia de patrones de comunicación que se encuentran preestablecidos dentro de las organizaciones, los mismos que difieren ampliamente por la diversidad de cultura y grupos etarios, donde se fijan estratos y clases sociales que se proyectan como barreras para la interacción social.
Moreno et al. (2014) mencionan que para tener éxito en el ámbito laboral, es indispensable ser puntual, mientras que dicho aspecto en Venezuela es imprescindible, lo que conlleva a diferencias aspectos básicos en distintas partes del mundo, tales como Europa y Norteamérica donde la asertividad es catalogada como parte del buen estado o salud mental y su carencia como un importante generador de intranquilidad.

Por otra parte, Amigó Borrás y Hernández Flórez (2012), mencionan en relación a los factores de personalidad y felicidad que "una de las emociones positivas más importante es la felicidad" (p. 42) considerada como un estado transitorio que se caracteriza por la alegría y el júbilo del individuo. Ante aquello, dicho estado emocional conlleva a la persona a ser más asertiva y mejorar sus relaciones interpersonales.

Para Garcés Delgado, Santana Vega, y Feliciano García (2012) resulta importante establecer herramientas esenciales que permitan desarrollar efectivamente las habilidades sociales, para que de esta manera los seres humanos puedan relacionarse de manera asertiva con los demás. En este sentido, las personas que son atendidas por profesionales con habilidades sociales plenamente desarrolladas entran en un estado de satisfacción total que beneficia la imagen de la empresa prestadora, por ejemplo de algún servicio.

Ahora bien, mejorar las relaciones entre los trabajadores de una empresa puede potenciar de manera diferente la eficacia y eficiencia de cada uno de los individuos, 
aumentando de manera significativa su desempeño laboral y por ende el éxito empresarial (Lorenzo y Bueno, 2011). En este sentido, las habilidades sociales e interpersonales tienden a marcar la tendencia entre el surgimiento de las nuevas organizaciones con un amplio margen de diferenciación entre los contextos gerenciales tradicionales, ya que estas apuestan a la salud organizacional y cimenta la satisfacción laboral como un eje transversal que les permite alcanzar sus objetivos.

Al hablar de organizaciones saludables, es necesario hacer énfasis en el desarrollo adecuado de las habilidades sociales como resultado de la satisfacción laboral. Por ello, Giraldo y Sanín (2015) manifiestan que "la satisfacción laboral es uno de los contextos sociales que suele explorarse en el interior de las organizaciones debido a sus conocidos efectos sobre las condiciones que generan altos costos" (pág. 34) por lo que surge la importancia de los instrumentos que permiten conocer sus niveles. Bajo este contexto, la satisfacción laboral puede ser entendida como una reacción afectiva de agrado que se origina al contrastar la realidad del trabajo con las expectativas e intereses propios que convergen con dicha realidad. De este modo la satisfacción con el trabajo se proyecta como la realidad coincidente con las expectativas propias del individuo. La satisfacción se define como aquella sensación que el individuo experimenta al lograr el restablecimiento del equilibrio entre una necesidad o grupo de necesidades y el objeto o los fines que la reducen. Por tanto, esta es considerada como el producto de las actitudes que posee cada empleado con referencia a sus actividades laborales.

Shragay y Tziner (2011) manifiestan que: "los empleados pueden estar satisfechos con determinados aspectos del trabajo y con otros no, a la vez que determinan una valoración general que puede relacionarse con la satisfacción o insatisfacción" (pág. 65). En este sentido, existen criterios variados que tienden a comparar a la satisfacción con el estado de felicidad debido a que es un componente afectivo; sin embargo, estos hacen referencia a dos fenómenos totalmente distintos, lo que implica que esta no debe entenderse como un rasgo, en tanto mantiene una dependencia de las expectativas, las que se consideran cambiantes. Por otra parte, existe una distinción significativa inherente a la visión de temporalidad, donde la satisfacción depende directamente de las condiciones presentes, mientras que los estados de felicidad se relacionan con la acumulación de eventos positivos provenientes del pasado (Seligman, 2006). Por ello, los niveles bajos de satisfacción pueden ser entendidos como los altos niveles de insatisfacción que se asocian como un tipo de fenómenos de ausentismo, rotación o toma de decisiones de carácter colectivo, quejas, reclamos y evitación directa de la situación de trabajo que repercute en la productividad y eficiencia de la organización por los costos que estos representan. En caso contrario, la presencia de reacciones efectivas favorables acerca de los contextos laborales se relaciona de manera positiva en la presencia de una diversidad de comportamientos de 
quienes integran la organización, los mismos que determinan su compromiso, motivación e intensión de permanencia. Por ende, la satisfacción laboral hace parte de las medidas de bienestar y la satisfacción directa de las necesidades del individuo en todo su contexto.

Alonso (2008) asegura que la satisfacción laboral es un constructo pluridimensional que depende tanto de las características individuales del sujeto como de las características y especificidades del trabajo que realiza. Además, el concepto de satisfacción en el trabajo está integrado por un conjunto de satisfacciones específicas, o aspectos parciales, que determinan la satisfacción general. Así entendida, la satisfacción laboral es una reacción afectiva general de una persona en relación con todos los aspectos del trabajo y del contexto laboral; es una función de todas las facetas parciales de la satisfacción. De acuerdo a Maslow (1991) las personas que no llegan a cubrir las necesidades básicas tienden a tener un pésimo desarrollo personal y laboral, ya que viven en conflicto no solo con ellos mismos si no también con las personas que los rodean, impidiendo que exista una armonización personal, y una buena comunicación. Salessi y Omar (2017) destacan la importancia de las fortalezas psicológicas que conforman el constructo donde se encuentra la esperanza, auto eficiencia, optimismo y resiliencia que convergen en los aspectos de bienestar y calidad de vida. A partir de ello, se establece una relación directa con la satisfacción laboral de los trabajadores en un nivel superior a cualquiera de sus componentes individuales. Por tanto, existe un renovado interés por conocer las nuevas tendencias sociales y organizacionales que inciden en la satisfacción laboral. En este sentido, Meza Barajas (2017) a través del análisis de la percepción del género entre clima organizacional y satisfacción laboral del sector industrial, asegura que el rol de los trabajadores en un determinado contexto de trabajo se ve influenciado directamente por la propia organización, quien es capaz de incidir en su desempeño y permanencia por medio de factores con características intrínsecas al individuo para mantener la satisfacción y el clima acorde para que puedan ser percibidos de formas diferentes, especialmente por la especificación del género.

Ante lo mencionado, Pujol-Cols y Dabos (2018) al estudiar la satisfacción laboral ponen de manifiesto el nivel de influencia que las variables organizacionales y los factores situacionales intrínsecos y extrínsecos ejercen sobre el comportamiento, actitudes y estados de bienestar del personal, los que están relacionados con: las recompensas justas, el salario digno, desafío laboral, condiciones laborales, condiciones favorables, afinidad entre equipo de trabajo, ascensos, trabajo en equipo, entre otros. Por ello, dentro de las organizaciones es imprescindible que el trabajo en equipo se encuentre enfocado al cumplimiento de los objetivos empresariales como un medio para su satisfacción personal (Alonso, 2008).

Paz y Cubo (2017) considera que: "la satisfacción laboral es el extracto de 
actitudes de los individuos al desempeñar sus tareas" (pág. 83), es por ello que, si un trabajador está complacido con su lugar de trabajo pondrá una buena predisposición en sus deberes laborales, mostrando un positivismo que dará resultados eficaces, lo que por el contrario no sucederá si la situación fuera inversa.

De la misma manera Cajas et al (2017) aseguran que el éxito de las organizaciones modernas se centra en la capacidad de contar con colaboradores satisfechos, para lo cual es necesario proporcionar un ambiente de trabajo que se enfoque en su satisfacción laboral, las mismas que son cambiantes como parte de las condicionantes de los medios en que se desenvuelve (Haz y Fierro, 2017). De manera adicional, autores como Rosa, Navarro-Segura y López (2014) consideran que la satisfacción laboral, es el producto de un adecuado desarrollo de las habilidades sociales por parte del personal colaborativo, quienes contribuyen de manera efectiva a la consolidación de los objetivos empresariales. En este sentido, las relaciones interpersonales de cada uno de ellos favorecen al bienestar y clima laboral, lo que implica la presencia de trabajadores complacidos y satisfechos, plenamente productivos. Lacunza (2012) expresa la necesidad de reconocer la relevancia de las habilidades sociales y la relación directa con la satisfacción laboral dentro de las empresas, se puede mencionar que existe un vínculo con la competitividad y el éxito. Garcés Delgado, Santana Vega, y Feliciano García (2012) concuerdan en que: "la cohesión entre las habilidades sociales, la satisfacción laboral y por ende la productividad de una empresa dependerá de una homeostasis laboral de estas dos variables" (pág. 65).

Las empresas que tienen una cultura de trabajo con visión estratégica, consideran que la satisfacción de sus colaboradores, permite alcanzar los logros de la empresa, convirtiendo al bienestar laboral en un eje transversal en sus operaciones diarias, siendo el sistema de compensaciones, las condiciones de trabajo tanto físicas como psicológicas, así como el desarrollo del personal, elementos importantes de satisfacción (Alvarez, López, \& Silva, 2019). Ante aquello, las organizaciones deben enfatizar sus acciones para mejorar la satisfacción laboral de sus colaboradores, teniendo presente varios componentes, entre los que se encuentran: la diversidad de habilidades, exactitud de las tareas encomendadas, representación de la tarea, autonomía y retroalimentación del puesto (Tapia y Cubo, 2017).

\section{Metodología}

Se realizó un estudio cuantitativo correlacional de tipo transaccional, con una muestra no probabilística, en la que se consideraron 256 trabajadores residentes en Milagro con al menos un año de experiencia laboral, con edades comprendidas entre los 18 y los 69 años con una media de 31,36 años. La distribución por antigüedad fluctúa entre 1 y 30 años de trabajo con una media de 4,63 años y laboran en diferentes áreas de trabajo, con mayor énfasis en el área de servicios. 
Como instrumento se utilizó la Escala de Habilidades Sociales -EHS (Gismero, 2010), el cual evalúa 6 factores: Autoexpresión en situaciones sociales, defensa de los propios derechos como consumidor, expresión de enfado o disconformidad, decir no y cortar interacciones, hacer peticiones e iniciar interacciones positivas con el sexo opuesto; contiene 33 preguntas con escala de medición Likert de 4 opciones (Opción A: No me identifico, en la mayoría de las veces no me ocurre o no lo haría; B: No tiene que ver conmigo, aunque alguna vez me ocurra; $\mathrm{C}$ : $\mathrm{Me}$ describe aproximadamente, aunque no siempre actúe así o me sienta así, y D: Muy de acuerdo, me sentiría así o actuaría así en la mayoría de los casos). Para medir la Satisfacción Laboral se utilizó el instrumento SL10/12 (Meliá y Peiró, 1998), el cual consta de 12 ítems, medidos con escala Likert de 7 puntos (1: Muy Insatisfecho, 2: Bastante Insatisfecho, 3: Algo insatisfecho, 4:
Indiferencia, 5: Algo Satisfecho, 6: Bastante Satisfecho, y 7: Muy Satisfecho).

Se realizó el análisis estadístico descriptivo y correlacional y se aplicó regresión lineal con el apoyo del paquete estadístico SPSS versión 22. En el análisis de fiabilidad de la escala de Satisfacción se obtuvo un a de Cronbach de 0,93 y para la de Habilidades Sociales fue de 0,89 .

\section{Resultados}

En cuanto a la satisfacción laboral se observa en la Figura 1 que los participantes priorizan el entorno físico de su lugar de trabajo y su limpieza, salubridad e higiene del lugar, lo cual está acorde con el estudio realizado en la provincia de Esmeraldas- Ecuador en personal de salud, en el que se obtuvo una relación significativa entre el clima organizacional y la satisfacción laboral
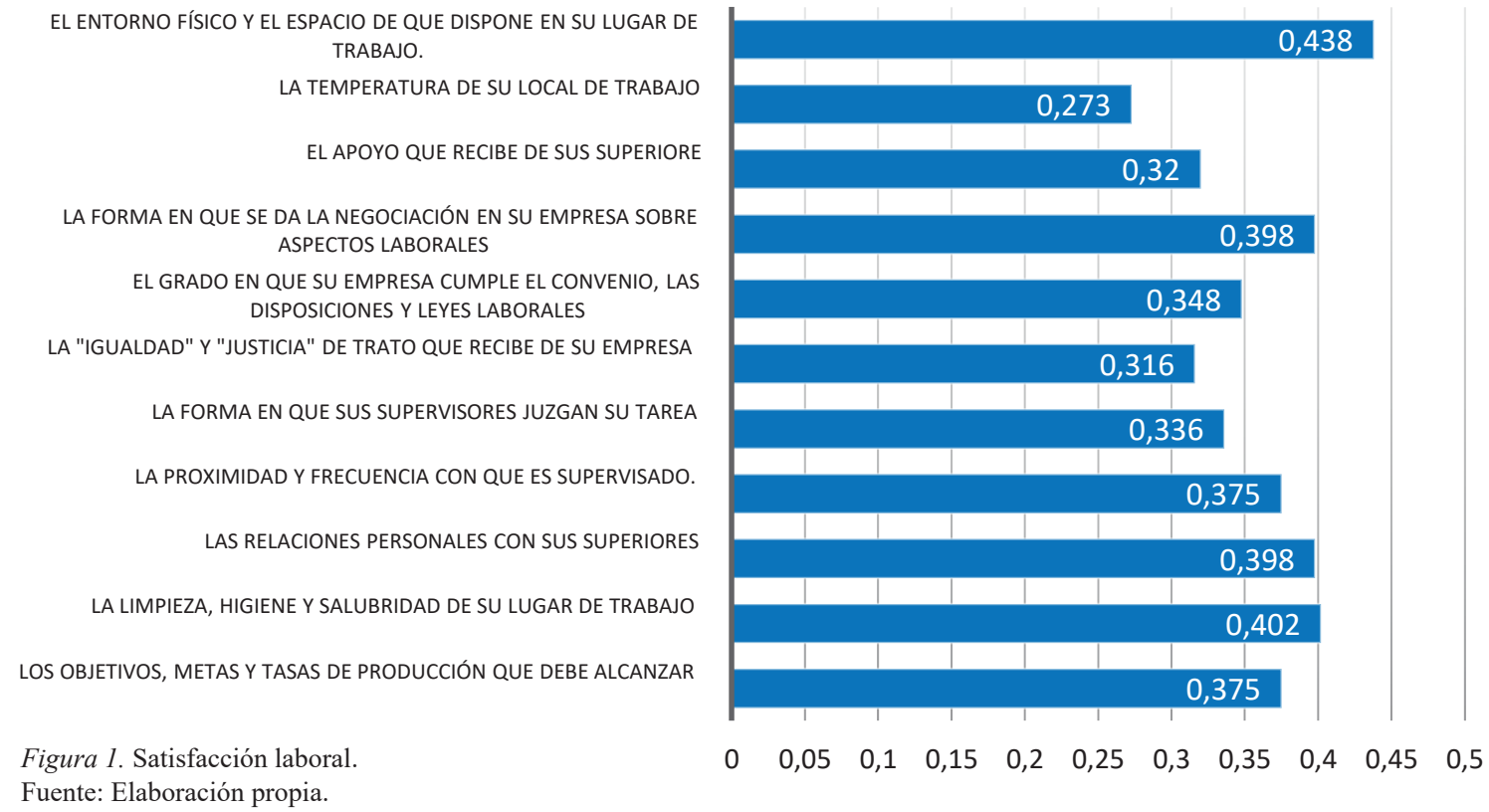


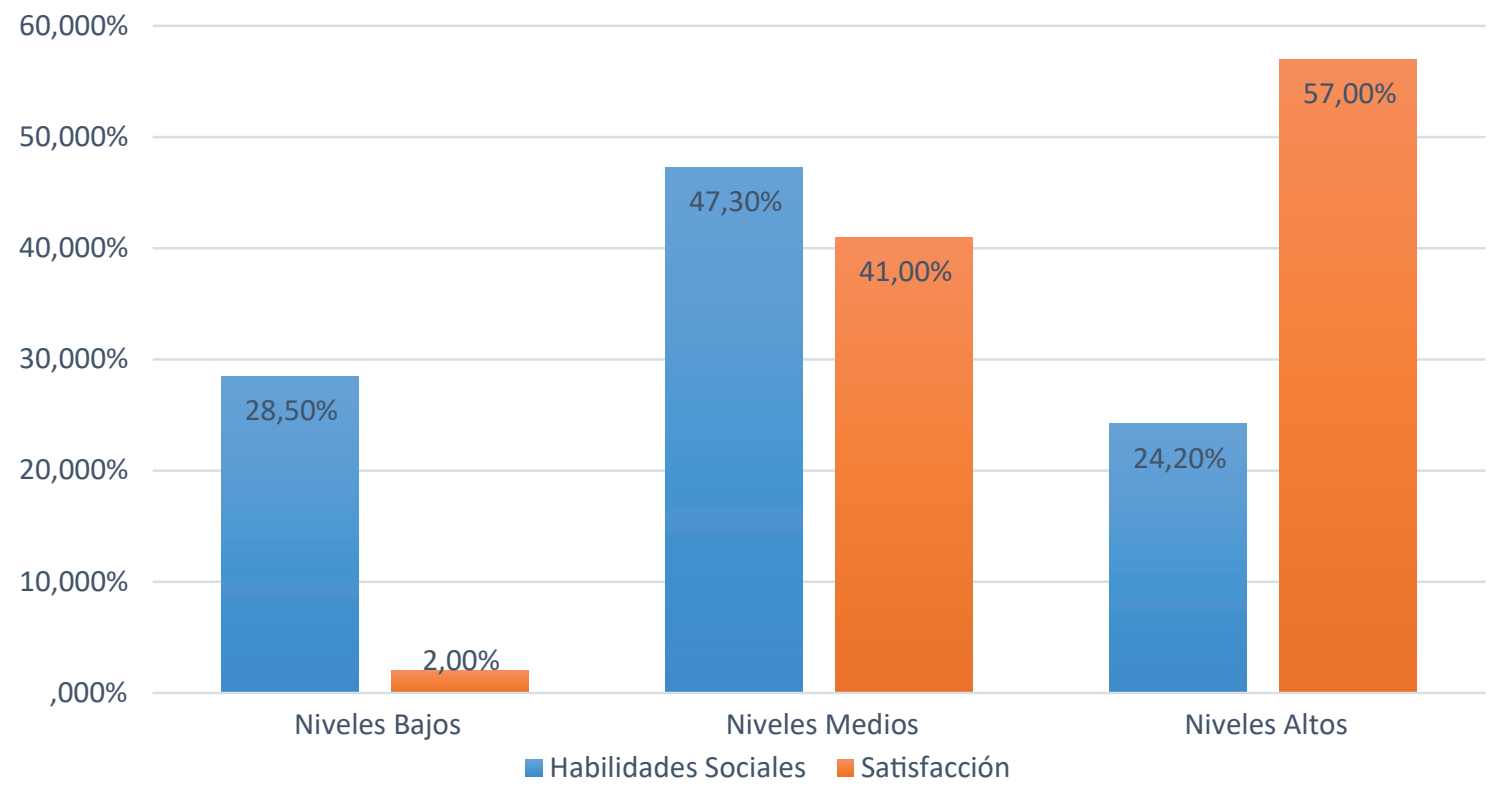

Figura 2. Niveles de Habilidades Sociales y Satisfacción.

Fuente: Elaboración propia.

(Manosalvas, Manosalvas, y Nieves, 2015).

Destacan entre los resultados los niveles altos de satisfacción con un 57\% y las habilidades sociales se encuentran en un nivel medio con el 47,30\% (ver Figura 2).

En la Tabla 1 se presentan las correlaciones estadísticamente significativas. Se observa que casi todas las correlaciones son negativas. Se encontró que existe una correlación significativa entre el estado civil y la importancia que le otorga al apoyo que recibe de sus jefes; dependiendo el área de trabajo en la que se labora existirá una correlación negativa con la temperatura del lugar, coincidiendo con los estudios realizados por Álvarez, López y Silva (2019).

En cuanto a la relación entre las habilidades sociales y la satisfacción en el trabajo se concluyó que una persona con dificultad para auto expresarse considera como elementos de satisfacción, el alcanzar los objetivos y metas de producción, es así como Deming (2017), indica que las habilidades sociales mejoran la capacidad de coordinación, y por lo tanto, la factibilidad de conseguir objetivos. También es necesario mencionar que quienes tienen dificultad para auto expresarse otorgan importancia al tipo de supervisión que ejercen sobre él, a la igualdad y justicia de trato que recibe de su empresa y el apoyo de sus superiores. La dificultad de hacer peticiones produce insatisfacción en el cumplimiento de los objetivos y metas, por lo que valoran mucho el tipo de supervisión que ejercen, coincidiendo con Cajas et al (2017) quienes consideran que el tipo de dirección es una causa de satisfacción laboral; por otra parte, la proximidad y frecuencia en la que es supervisado y la forma como es juzgada 
Tabla 1

Matriz de Correlaciones: Relaciones Interpersonales vs. Satisfacción Laboral

\begin{tabular}{|c|c|c|c|c|c|c|c|c|}
\hline FACTORES & $\begin{array}{c}\text { LOS } \\
\text { OBJETIVOS, } \\
\text { METAS Y } \\
\text { TASAS DE } \\
\text { PRODUCCIÓN } \\
\text { QUE DEBE } \\
\text { ALCANZAR }\end{array}$ & $\begin{array}{c}\text { LA } \\
\text { TEMPERATUR } \\
\text { A DE SU } \\
\text { LOCAL DE } \\
\text { TRABAJO }\end{array}$ & $\begin{array}{c}\text { LA } \\
\text { SUPERVISIÓN } \\
\text { QUE } \\
\text { EJERCEN } \\
\text { SOBRE USTED }\end{array}$ & $\begin{array}{c}\text { LA } \\
\text { PROXIMIDAD } \\
\text { Y } \\
\text { FRECUENCIA } \\
\text { CON QUE ES } \\
\text { SUPERVISADO }\end{array}$ & $\begin{array}{c}\text { LA FORMA } \\
\text { EN QUE SUS } \\
\text { SUPERVISORES } \\
\text { JUZGA SU } \\
\text { TAREA }\end{array}$ & $\begin{array}{c}\text { LA } \\
\text { "IGUALDAD" } \\
\text { Y "JUSTICIA" } \\
\text { DE TRATO } \\
\text { QUE RECIBE } \\
\text { DE SU } \\
\text { EMPRESA }\end{array}$ & $\begin{array}{c}\text { ELAPOYO } \\
\text { QUE RECIBE } \\
\text { DE SUS } \\
\text { SUPERIORES }\end{array}$ & $\begin{array}{c}\text { LA FORMA EN } \\
\text { QUE SE DA LA } \\
\text { NEGOCIACIÓ } \\
\text { EN SU } \\
\text { EMPRESA } \\
\text { SOBRE } \\
\text { ASPECTOS } \\
\text { LABORALES }\end{array}$ \\
\hline Edad & & & & & & & &,- 173 \\
\hline Estado Civil & & & & & & &,$- 133^{\prime}$ & \\
\hline Área de trabajo & &,$- 147^{\prime}$ & & & &,$- 129^{\prime}$ & & \\
\hline $\begin{array}{l}\text { Autoexpresión } \\
\text { de situaciones } \\
\text { sociales }\end{array}$ &,- 139 & &,$- 126^{\prime}$ & & &,$- 143^{\prime}$ &,- 130 & \\
\hline $\begin{array}{l}\text { Hacer } \\
\text { peticiones }\end{array}$ &,$- 165^{\prime}$ & &,$- 152^{\prime}$ &,- 183 &,$- 127^{\prime}$ & & & \\
\hline $\begin{array}{l}\text { Iniciar } \\
\text { interacciones } \\
\text { positivas con } \\
\text { sexo opuesto }\end{array}$ & & &,$- 145^{\prime}$ & &,- 150 & & & \\
\hline
\end{tabular}

su tarea. De igual manera, la dificultad para establecer relaciones con el sexo opuesto se vincula con el tipo de supervisión y la forma como juzgan su tarea, esta ansiedad puede provocar un mal servicio, al respecto Luján et al (2012) mencionan que las habilidades sociales desarrolladas pueden ser causa de satisfacción de los usuarios.

\section{Conclusiones}

Las habilidades sociales se convierten en el primer paso para buscar soluciones que favorezcan a los colaboradores y a las instituciones, situación que influye en los niveles de satisfacción o insatisfacción, coincidiendo con Moreno et al (2014), quienes consideran que es necesario el desarrollo de las habilidades sociales para contribuir a mantener organizaciones saludables.
Una de las limitaciones presentadas se relaciona con la apertura de las instituciones para otorgar los permisos para realizar este tipo de investigaciones, motivo por el cual se direccionó hacia una muestra no cautiva, sin embargo, para futuros estudios sería conveniente incorporar un tercer instrumento que permita confrontar los resultados obtenidos con el nivel de clima laboral que exista en la organización evaluada.

\section{Referencias}

Alonso, P. (2008). Estudio comparativo de la satisfacción laboral en el personal. Revista de Psicología del Trabajo y de las Organizaciones, 24(1), 25-40.

Álvarez, L. A., López, L., y Silva, N. (2019). Satisfacción laboral en una organización human care de la Bahía de Guayaquil. PODIUM, 35, 87-96. 
Amigó Borrás, S., y Hernández Flórez, N. (2012). Factor general de personalidad y felicidad : Un estudio desde la perspectiva rasgo-estado en una muestra colombiana. Pensando Psicología, 8(14), 1-11.

Berman, E., y West, J. (2008). Managing Emotional Intelligence in U.S. Cities: A Study of Social Skills among Public Managers. Public Administration Review, 68(4), 742-758.

Caballo, V. (1983). Assertivity: Definitions and dimensions. Estudios de Psicología, 4(13), 51-62.

doi:https://doi.org/10.1080/02109395.19 83.10821343

Caballo, V. (2007). Manual de evaluación y entrenamiento de las habilidades sociales. Madrid: Editores Siglo XXI de España, S.A.

Cajas, M., Gastiaburo, J., Espinel , A., Espinel, J., y Ruperti, E. (2017). Factores de Satisfacción e Insatisfacción laboral en trabajadores de la Ciudad de Milagro. Psicología Unemi, 1(1), 15-23.

Cornelius, H., y Faire, S. (2017). Tu ganas, yo gano: Como resolver conflictos creativamente. Madrid: Gaia Ediciones.

Corredor, G., Justicia- Arráez, A., Romero-López, M., \& Benavides-Nieto, A. (2017). Longitudinal Study of the Effects of Social Competence on Behavioral Problems. Procedia - Social and Behavioral Sciences, 237, 479-485.

Del Prette, Z., Monzani da Rocha, M., de Matos Silvares, E., y Del Prette, A. (2012). Social skills and psychological disorders: Converging and criterion-related validity for YSR and IHSA. Universitas Pshychologica, 11(3), 941-955.

Deming, D. J. (2017). The Growing Importance of Social Skill in the Labor Market. Quarterly Journal of Economics, 132(4), 1593-1640.
Patrício Do Amaral, M., Maia Pinto, F., y Bezerra de Medeiros, C. (2015). Las habilidades sociales y el comportamiento infractor en la adolescencia. Subjetividad y Procesos Cognitivos, 19(2), 17-38.

Eceiza, M., Arrieta, M., y Goñi, A. (2008). Habilidades sociales y contextos de la conducta social. Revista de Psicodidáctica, 13(1), 11-26.

Ferris, G., Treadway, D., y Perrewé, P. (2007). Political Skill in Organizations. Journal of Management, 33(3), 290-320. DOI: $10.1177 / 0149206307300813$

Flores Mamaní, E., García Tejada, M., Calsina Ponce, W., y Yapuchura Sayco, A. (2016). Las habilidades sociales y la comunicación interpersonal de los estudiantes de la Universidad del Altiplano-Puno. Comunic@cción, 7(2), 5-14.

Garcés Delgado, Y., Santana Vega, L., y Feliciano García, L. (2012). El desarrollo de habilidades sociales: una estrategia para potenciar la integración de menores en riesgo de exclusión. Revista Electrónica de Investigación y Docencia, 172-191.

García Esparza, A., \& Méndez Sánchez, C. (2017). El entrenamiento en habilidades sociales y su impacto en la convivencia escolar dentro de un grupo de primaria. Revista de Estudios y Experiencias En Educación, 16(30), 151-164.

Giraldo, M., y Sanín, A. (2015). Actualización Psicométrica de la Escala de Satisfacción Laboral SAT II. Revista Interamericana de Psicología Ocupacional, 33(2), 85-93.

Gismero, E. (2010). Manual de la Escala de Habilidades Sociales (3a ed.). Madrid: TEA, Ediciones, S.A.

Haz Vidal, I., y Fierro Ulloa, I. (2017). Liderazgo y Satisfacción Laboral: Un Análisis de Estudios Previos. PODIUM, 29-40.

Lacunza, A. (2012). Las intervenciones en habilidades 
sociales: revisión y análisis desde una mirada salugénica. Psicodebate. Psicología, Cultura y Sociedad, 12, 63-84.

Lorenzo, M., y Bueno, M. (2011). Entrenamiento De Habilidades Sociales En Fútbol Base: Propuesta De Intervención. SOCIOTAM, 21(2), 39-53.

Luján, I., Leal, C., Gascón, M. J., Espadas, M. J., y van der Hofstadt, C. (2012). Habilidades sociales en los profesionales de la salud y satisfacción de los pacientes del hospital de día polivalente de un hospital comarcal. Revista de Psicología de la Salud, 24(1), 127-141.

Manosalvas, C, Manosalvas, L., y Nieves, J. (2015). El clima organizacional y la satisfacción laboral: Un análisis cuantitativo riguroso de su relación. Ad-Minister, 26, 5-15.

Maslow, A. (1991). Motivación y personalidad. Madrid: Ediciones Díaz de Santos.

Meliá, J. L., y Peiró, J. M. (1998). Cuestionario de satisfaccion laboral S10/12. Psicologia de la seguridad laboral, 2-4.

Meza Barajas, E. (2017). Análisis en la percepción del género entre clima organizacional y satisfacción laboral del sector industrial-México.Comunic@cción, $8(2), 148-158$.

Moreno, B., Blanco, L., Aguirre, A., de Rivas, S., y Herrero, M. (2014). Habilidades sociales para las nuevas organizaciones. Psicología Conductual = behavioral psychology: Revista internacional de psicología clínica y de la salud, 22(3), 585-602.

Rosa, G., Navarro-Segura, L., y López, P. (2014). El Aprendizaje de las Habilidades Sociales en la Universidad. Análisis de una Experiencia Formativa en los Grados de Educación Social y Trabajo Social. Revista de formación universitaria, 7(4), 25-38.
Ovejero, A. (1990). Las Habilidades Sociales y su Entrenamiento; un Enfoque necesariamente Psicosocial. Psicothema, 2(2), 93-112.

Paz, C., y Cubo, S. (2017). Habilidades sociales relevantes: percepciones de múltiples actores educativos. Magis. Revista Internacional de Investigación en Educación, 9(19), 133-148.

Pujol-Cols, L., y Dabos, G. (2018). Satisfacción laboral: una revisión de la literatura acerca de sus principales determinantes. Journal of Management and Economics for Iberoamerica, 34(146), 3-18.

Rodríguez Matamoros, L. Y., Cacheiro González, M. L., \& Gil Pascual, J. A. (2014). Desarrollo De Habilidades Sociales En Estudiantes. Desarrollo de Habilidades Sociales En Estudiantes Mexicanos de Preparatoria a Través de Actividades Virtuales En Plataforma Moodle. TESI, 15(3), 1-255.

Salessi, S., y Omar, A. (2017). Satisfacción Laboral: Un Modelo Explicativo Basado en Variables Disposicionales. Revista Colombiana de Psicologia, 26(2), 329-345. https://doi.org/10.15446/rcp.v26n2.60651

Seligman, M. (2006). La evolución y el sentimiento positivo. Barcelona: Ediciones B, S. A.

Shragay, D., y Tziner, A. (2011). El Efecto Generacional sobre la Relación entre Implicación en el Puesto, Satisfacción Laboral y Conducta Cívica. Revista de Psicología del Trabajo y de las Organizaciones, 27(2), 143-157.

Tapia, C., y Cubo, S. (2017). Habilidades sociales relevantes: percepciones de múltiples actores educativos. Magis Revista Internacional de Investigación en Educación, 9(19), 133-148.

Zavala Berbena, M., Valadez Sierra, M., \& Vargas 
Vivero, M. (2008). Inteligencia emocional y habilidades sociales en adolescentes con alta aceptación social. Electronic Journal of Research in Educational Psychology, 6(2), 321-338.

PODIUM No. 36, Diciembre 2019, pp. 89-102

(C) Universidad Espíritu Santo - UEES

ISSN: 1390-5473 e-ISSN: 2588-0969 